\title{
QUASILINEAR DEGENERATE ELLIPTIC UNILATERAL PROBLEMS
}

L. AHAROUCH, Y. AKDIM, AND E. AZROUL

Received 20 February 2004

We will be concerned with the existence result of a degenerate elliptic unilateral problem of the form $A u+H(x, u, \nabla u)=f$, where $A$ is a Leray-Lions operator from $W^{1, p}(\Omega, w)$ into its dual. On the nonlinear lower-order term $H(x, u, \nabla u)$, we assume that it is a Carathéodory function having natural growth with respect to $|\nabla u|$, but without assuming the sign condition. The right-hand side $f$ belongs to $L^{1}(\Omega)$.

\section{Introduction}

Let $\Omega$ be a bounded open set of $\mathbb{R}^{N}$, let $p$ be a real number such that $1<p<\infty$, and let $w=$ $\left\{w_{i}(x), 0 \leq i \leq N\right\}$ be a vector of weight functions on $\Omega$, that is, each $w_{i}(x)$ is a measurable a.e. strictly positive function on $\Omega$, satisfying some integrability conditions. And let $A u=$ $-\operatorname{div}(a(x, u, \nabla u))$ be a Leray-Lions operator defined from the weighted Sobolev space $W_{0}^{1, p}(\Omega, w)$ into its dual $W^{-1, p^{\prime}}\left(\Omega, w^{*}\right)$.

The aim of this paper is to study the degenerate unilateral problems associated to a nonlinear operator of the form

$$
\begin{gathered}
-\operatorname{div}(a(x, u, \nabla u))+H(x, u, \nabla u)=f \quad \text { in } \Omega, \\
u=0 \quad \text { on } \partial \Omega,
\end{gathered}
$$

where $H$ is a nonlinear lower-order term having natural growth with respect to $|\nabla u|$. With respect to $|u|$ we do not assume any growth restrictions (i.e., $H(x, s, \xi) \leq \gamma(x)+$ $\left.g(s) \sum_{i=1}^{N} w_{i}\left|\xi_{i}\right|^{p}\right)$. The right-hand side $f$ belongs to $L^{1}(\Omega)$. More precisely, we prove the existence of solutions for the following nonlinear Dirichlet problems:

$$
\begin{gathered}
u \geq \psi \quad \text { a.e. in } \Omega, \\
T_{k}(u) \in W_{0}^{1, p}(\Omega, w) \quad \forall k>0, \\
\int_{\Omega}(a(x, u, \nabla u)) T_{k}(u-v)+\int_{\Omega} H(x, u, \nabla u) T_{k}(u-v) \\
\leq \int_{\Omega} f T_{k}(u-v) \quad \forall v \in K_{\psi} \cap L^{\infty}(\Omega),
\end{gathered}
$$


where $K_{\psi}=\left\{u \in W_{0}^{1, p}(\Omega, w), u \geq \psi\right.$ a.e. in $\left.\Omega\right\}$ and $T_{k}$ is the usual truncation operator. Note that the existence result is proved without assuming the sign condition $H(x, s, \xi)$ $s \geq 0$. For that we prove the strong convergence of the truncations $T_{k}\left(u_{n}\right)$ in $W_{0}^{1, p}(\Omega, w)$, where $u_{n}$ is a solution of the approximate problem. If we take $\psi=-\infty$, we obtain the existence result of problem (1.1) in the case of equation.

Recently in [9] Porretta studied problem (1.1) in the classical Sobolev space $W_{0}^{1, p}(\Omega)$ where the right-hand side is a measure. We point out that another work in this direction can be found in [6] where problem (1.1) is studied with $f \in L^{m}(\Omega)$, for which the authors have proved that there exists a bounded weak solution for $m>(N / 2)$, and an unbounded entropy solution for $(N / 2)>m>(2 N /(N+2))$. A different approach (without using the sign condition) was introduced also in [5] when $b(x, s, \xi)=\lambda s-|\xi|^{2}$ with $\lambda>0$. Our paper can be seen as a generalization of [9] in the weighted case and as a continuation of $[1,2,3,4]$ where in [1] the case of degenerate variational equation is treated and in $[2,3]$ the variational degenerate inequalities are studied, while in [4] the authors were concerned with problem (1.1) with the right-hand side $f$ assumed to belong either to $W^{-1, p^{\prime}}\left(\Omega, w^{*}\right)$ or to $L^{1}(\Omega)$. In $[1,2,3,4]$ we suppose that the lower-order term satisfies the sign condition $H(x, s, \xi) s \geq$ for all $s \in \mathbb{R}$, while in [4] (where $f \in L^{1}(\Omega)$ ), we have assumed also the exact natural growth, that is, $|H(x, s, \xi)| \geq \rho \sum_{i=1}^{N} w_{i}\left|\xi_{i}\right|^{p}$.

\section{Preliminaries}

Let $\Omega$ be a bounded open subset of $\mathbb{R}^{N}(N \geq 1)$. Let $1<p<\infty$, and let $w=\left\{w_{i}(x) ; i=\right.$ $1, \ldots, N\}, 0 \leq i \leq N$, be a vector of weight functions, that is, every component $w_{i}(x)$ is a measurable function which is strictly positive a.e. in $\Omega$. Further, we suppose in all our considerations that, for $0 \leq i \leq N$,

$$
w_{i} \in L_{\mathrm{loc}}^{1}(\Omega), \quad w_{i}^{-1 /(p-1)} \in L_{\mathrm{loc}}^{1}(\Omega)
$$

We define the weighted space with weight $\gamma$ in $\Omega$ as

$$
L^{p}(\Omega, \gamma)=\left\{u(x), u \gamma^{1 / p} \in L^{p}(\Omega)\right\}
$$

which is endowed with the norm

$$
\|u\|_{p, \gamma}=\left(\int_{\Omega}|u(x)|^{p} \gamma(x) d x\right)^{1 / p}
$$

We denote by $W^{1, p}(\Omega, w)$ the space of all real-valued functions $u \in L^{p}\left(\Omega, w_{0}\right)$ such that the derivatives in the sense of distributions satisfy

$$
\frac{\partial u}{\partial x_{i}} \in L^{p}\left(\Omega, w_{i}\right) \quad \forall i=1, \ldots, N
$$


This set of functions forms a Banach space under the norm

$$
\|u\|_{1, p, w}=\left(\int_{\Omega}|u(x)|^{p} w_{0} d x+\sum_{i=1}^{N} \int_{\Omega}\left|\frac{\partial u}{\partial x_{i}}\right|^{p} w_{i}(x) d x\right)^{1 / p}
$$

To deal with the Dirichlet problem, we use the space

$$
X=W_{0}^{1, p}(\Omega, w)
$$

defined as the closure of $C_{0}^{\infty}(\Omega)$ with respect to the norm (2.5). Note that $C_{0}^{\infty}(\Omega)$ is dense in $W_{0}^{1, p}(\Omega, w)$ and $\left(X,\|\cdot\|_{1, p, w}\right)$ is a reflexive Banach space.

We recall that the dual space of the weighted Sobolev spaces $W_{0}^{1, p}(\Omega, w)$ is equivalent to $W^{-1, p^{\prime}}\left(\Omega, w^{*}\right)$, where $w^{*}=\left\{w_{i}^{*}=w_{i}^{1-p^{\prime}}\right\}, i=1, \ldots, N$, and $p^{\prime}$ is the conjugate of $p$, that is, $p^{\prime}=p /(p-1)$. For more details we refer the reader to [7].

\section{Main general results}

3.1. Basic assumptions and statement of result. We state the following assumptions.

$\left(H_{1}\right)$ The expression

$$
\|\mid u\| \|_{X}=\left(\sum_{i=1}^{N} \int_{\Omega}\left|\frac{\partial u}{\partial x_{i}}\right|^{p} w_{i}(x) d x\right)^{1 / p}
$$

is a norm defined on $X$ and is equivalent to the norm (2.5). Note that $\left(X,\|u\|_{X}\right)$ is a uniformly convex (and reflexive) Banach space.

$\left(H_{2}\right)$ There exist a weight function $\sigma$ on $\Omega$ and a parameter $q$, satisfying

$$
\begin{gathered}
1<q<p+p^{\prime}, \\
\sigma^{1-q^{\prime}} \in L_{\mathrm{loc}}^{1}(\Omega),
\end{gathered}
$$

with $q^{\prime}=q /(q-1)$ and such that the Hardy inequality

$$
\left(\int_{\Omega}|u|^{q} \sigma(x) d x\right)^{1 / q} \leq C\left(\sum_{i=1}^{N} \int_{\Omega}\left|\frac{\partial u}{\partial x_{i}}\right|^{p} w_{i}(x) d x\right)^{1 / p}
$$

holds for every $u \in X$ with a constant $C>0$ independent of $u$. Moreover, the imbedding

$$
W_{0}^{1, p}(\Omega, w) \hookrightarrow \hookrightarrow L^{q}(\Omega, \sigma)
$$

determined by inequality (3.4) is compact. 
14 Quasilinear degenerate elliptic unilateral problems

On the other hand, we consider the nonlinear elliptic differential operator in divergence form, defined from $W_{0}^{1, p}(\Omega, w)$ into its dual $W^{-1, p^{\prime}}\left(\Omega, w^{*}\right)$ by

$$
A u=-\operatorname{div}(a(x, u, \nabla u))
$$

where $a: \Omega \times \mathbb{R} \times \mathbb{R}^{N} \rightarrow \mathbb{R}^{N}$ is a Carathéodory function satisfying the following conditions.

For all $s \in \mathbb{R}, \xi, \eta \in \mathbb{R}^{N}$ and for almost every $x \in \Omega$,

$$
\begin{gathered}
\left|a_{i}(x, s, \xi)\right| \leq \beta w_{i}^{1 / p}(x)\left[k(x)+\sigma^{1 / p^{\prime}}|s|^{q / p^{\prime}}+\sum_{j=1}^{N} w_{j}^{1 / p^{\prime}}(x)\left|\xi_{j}\right|^{p-1}\right] \text { for } i=1, \ldots, N, \\
{[a(x, s, \xi)-a(x, s, \eta)](\xi-\eta)>0 \quad \forall \xi \neq \eta} \\
a(x, s, \xi) \xi \geq \alpha \sum_{i=1}^{N} w_{i}(x)\left|\xi_{i}\right|^{p}
\end{gathered}
$$

where $k(x)$ is a positive function in $L^{p^{\prime}}(\Omega)$ and $\alpha, \beta$ are positive constants.

Furthermore, let $H(x, s, \xi): \Omega \times \mathbb{R} \times \mathbb{R}^{N} \rightarrow \mathbb{R}$ be a Carathéodory function such that for a.e. $x \in \Omega$ and for all $s \in \mathbb{R}, \xi \in \mathbb{R}^{N}$, the growth condition

$$
|H(x, s, \xi)| \leq \gamma(s)+g(s) \sum_{i=1}^{N} w_{i}(x)\left|\xi_{i}\right|^{p}
$$

is satisfied, where $g: \mathbb{R} \rightarrow \mathbb{R}^{+}$is a continuous positive function that belongs to $L^{1}(\mathbb{R})$, while $\gamma(x)$ belongs to $L^{1}(\Omega)$.

Finally, let the convex set

$$
K_{\psi}=\left\{u \in W_{0}^{1, p}(\Omega, w), u \geq \psi \text { a.e. in } \Omega\right\}
$$

We will prove the following existence theorem.

Theorem 3.1. Assume that the assumptions $\left(H_{1}\right),\left(H_{2}\right)$, and (3.7)-(3.11) hold and let $f$ belong to $L^{1}(\Omega)$. Then, there exists a measurable function $u$ which is a solution of the following problem:

$$
\begin{gathered}
u>\psi \quad \text { a.e. in } \Omega, \\
T_{k}(u) \in W_{0}^{1, p}(\Omega, w), \\
\int_{\Omega} a(x, u, \nabla u) \nabla T_{k}(u-v) d x+\int_{\Omega} H(x, u, \nabla u) T_{k}(u-v) d x \\
\leq \int_{\Omega} f T_{k}(u-v) d x \quad \forall v \in K_{\psi} \cap L^{\infty}(\Omega) \forall k>0 .
\end{gathered}
$$

Remark 3.2. The statement of Theorem 3.1 generalizes in the weighted case the analogous one in [9] with $\mu \in L^{1}(\Omega)$ (where the case of equation is treated). 
Remark 3.3. We remark that in the case of $\psi=-\infty$, Theorem 3.1 gives the existence of solution in the case of equation, that is, the problem

$$
\begin{gathered}
T_{k}(u) \in W_{0}^{1, p}(\Omega, w), \quad k>0, \\
\int_{\Omega} a(x, u, \nabla u) \nabla T_{k}(u-v) d x+\int_{\Omega} H(x, u, \nabla u) T_{k}(u-v) d x \\
\leq \int_{\Omega} f T_{k}(u-v) d x \quad \forall v \in W_{0}^{1, p}(\Omega, w) \cap L^{\infty}(\Omega)
\end{gathered}
$$

has at least one solution.

3.2. Approximate problem. Let $\Omega_{n}$ be a sequence of compact subsets of $\Omega$ such that $\Omega_{n}$ is increasing to $\Omega$ as $n \rightarrow \infty$.

We consider the sequence of approximate problem

$$
\begin{gathered}
u_{n} \in K_{\psi}, \\
\int_{\Omega} a\left(x, u_{n}, \nabla u_{n}\right) \nabla\left(u_{n}-v\right) d x+\int_{\Omega} H_{n}\left(x, u_{n}, \nabla u_{n}\right)\left(u_{n}-v\right) d x \\
\leq \int_{\Omega} f_{n}\left(u_{n}-v\right) d x \quad \forall v \in K_{\psi},
\end{gathered}
$$

where $f_{n}$ are regular functions which strongly converge to $f$ in $L^{1}(\Omega)$ and $\left\|f_{n}\right\|_{L^{1}(\Omega)} \leq$ $\|f\|_{L^{1}(\Omega)}$ and

$$
H_{n}(x, s, \xi)=\frac{H(x, s, \xi)}{1+(1 / n)|H(x, s, \xi)|} \chi_{\Omega_{n}},
$$

where $\chi_{\Omega_{n}}$ is the characteristic function of $\Omega_{n}$.

Note that $\left|H_{n}(x, s, \xi)\right| \leq|H(x, s, \xi)|$ and $\left|H_{n}(x, s, \xi)\right| \leq n$.

Proposition 3.4. Assume that the assumptions $\left(H_{1}\right),\left(H_{2}\right)$, and (3.7)-(3.11) hold. Then, the problem $\left(\mathscr{P}_{n}\right)$ has at least one solution $u_{n} \in K_{\psi}$.

Proof. We define the operator $G_{n}: X \rightarrow X^{*}$ by

$$
\left\langle G_{n} u, v\right\rangle=\int_{\Omega} H_{n}(x, u, \nabla u) v d x .
$$

Thanks to Hölder's inequality, we have, for all $u \in X$ and all $v \in X$,

$$
\begin{aligned}
\left|\int_{\Omega} H_{n}(x, u, \nabla u) v d x\right| & \leq\left(\int_{\Omega}\left|H_{n}(x, u, \nabla u)\right|^{q^{\prime}} \sigma^{-q^{\prime} / q} d x\right)^{1 / q^{\prime}}\left(\int_{\Omega}|v|^{q} \sigma d x\right)^{1 / q} \\
& \leq n\left(\int_{\Omega_{n}} \sigma^{1-q^{\prime}} d x\right)^{1 / q^{\prime}}\|v\|_{q, \sigma} \\
& \leq c_{n} \mid\|v\| \| .
\end{aligned}
$$

The last inequality is due to (3.3) and (3.5). Consequently, in view of [3, Lemma 4.1], we deduce that the operator $B_{n}=A+G_{n}$ is pseudomonotone. 
On the other hand, we show that $B_{n}$ is coercive, in the following sense: there exists $v_{0} \in K_{\psi}$ such that

$$
\frac{\left\langle B_{n} v, v-v_{0}\right\rangle}{\|\mid v\|} \longrightarrow+\infty \quad \text { if }\|\mid v\| \| \longrightarrow \infty, v \in K_{\psi} .
$$

Let $v_{0} \in K_{\psi}$. From Hölder's inequality, the growth condition (3.7), and the compact imbedding (3.5), we have

$$
\begin{aligned}
\left\langle A v, v_{0}\right\rangle & =\sum_{i=1}^{N} \int_{\Omega} a_{i}(x, v, \nabla v) \frac{\partial v_{0}}{\partial x_{i}} d x \\
& \leq \sum_{i=1}^{N}\left(\int_{\Omega}\left|a_{i}(x, v, \nabla v)\right|^{p^{\prime}} w_{i}^{-p^{\prime} / p} d x\right)^{1 / p^{\prime}}\left(\int_{\Omega}\left|\frac{\partial v_{0}}{\partial x_{i}}\right|^{p} w_{i} d x\right)^{1 / p} \\
& \leq c_{1}||\left|v_{0}\right|||\left(\int_{\Omega} k(x)^{p^{\prime}}+|v|^{q} \sigma+\sum_{j=1}^{N}\left|\frac{\partial v}{\partial x_{j}}\right|^{p} w_{j} d x\right)^{1 / p^{\prime}} \\
& \leq c_{2}\left(c_{3}+\||| v||^{q / p^{\prime}}+\left.|||v|\right|^{p-1}\right),
\end{aligned}
$$

where $c_{i}$ are various constants.

Thanks to (3.9), we obtain

$$
\frac{\langle A v, v\rangle}{\|\mid v\| \|}-\frac{\left\langle A v, v_{0}\right\rangle}{\|\mid v\| \|} \geq \alpha\left\|\left|v\|\|^{p-1}-\left\|\left|v\|\|^{p-2}-\|\mid v\| \|^{\left(q / p^{\prime}\right)-1}-\frac{c}{\|\mid v\| \|} .\right.\right.\right.\right.
$$

In view of (3.2), we have $p-1>\left(q / p^{\prime}\right)-1$, then,

$$
\frac{\left\langle A v, v-v_{0}\right\rangle}{\|v\|} \longrightarrow \infty \quad \text { as }\|\mid v\| \| \rightarrow \infty .
$$

On the other hand,

$$
\begin{aligned}
\left|\left\langle G_{n} v, v\right\rangle\right| & =\left|\int_{\Omega_{n}} H_{n}(x, v, \nabla v) v d x\right| \\
& \leq\left(\int_{\Omega_{n}}\left|H_{n}(x, v, \nabla v)\right|^{q^{\prime}} \sigma^{-q^{\prime} / q} d x\right)^{1 / q^{\prime}}\left(\int_{\Omega_{n}}|v|^{q} \sigma d x\right)^{1 / q} \\
& \leq n\left(\int_{\Omega_{n}} \sigma^{-q^{\prime} / q} d x\right)^{1 / q^{\prime}}|| v \mid \| \\
& \leq c_{n}\||v|\|,
\end{aligned}
$$

hence (since $\left\langle G_{n} v, v\right\rangle /\||v|\|$ and $\left\langle G_{n} v, v_{0}\right\rangle$ are bounded), we have

$$
\frac{\left\langle B_{n} v, v-v_{0}\right\rangle}{\|\mid v\|}=\frac{\left\langle A v, v-v_{0}\right\rangle}{\||v|\|}+\frac{\left\langle G_{n} v, v\right\rangle}{\|\mid v\|}-\frac{\left\langle G_{n} v, v_{0}\right\rangle}{\|\mid v\|} \rightarrow \infty \quad \text { as }\|\mid v\| \| \rightarrow \infty .
$$

Finally, $B_{n}$ is pseudomonotone and coercive, hence by using [8, Chapter 2, Theorem 8.2], the approximate problem $\left(\mathscr{P}_{n}\right)$ has at least one solution. 


\subsection{A priori estimate}

Proposition 3.5. Assume that the assumptions $\left(H_{1}\right),\left(H_{2}\right)$, and (3.7)-(3.11) hold, and let $u_{n}$ be a solution of the approximate problem $\left(\mathscr{P}_{n}\right)$. Then, there exists a constant $c$ (which does not depend on the $n$ and $k$ ) such that

$$
\int_{\Omega} \sum_{i=1}^{N}\left|\frac{\partial T_{k}\left(u_{n}\right)}{\partial x_{i}}\right|^{p} w_{i} d x \leq c k,
$$

where $k>0$.

Proof. Let $v=u_{n}-\eta \exp \left(G\left(u_{n}\right)\right) T_{k}\left(u_{n}^{+}-\psi^{+}\right)$, where $\eta$ is a real positive and $G(s)=$ $\int_{0}^{s}(g(t) / \alpha) d t$ (note that the function $g$ is the one that appeared in (3.10)). Since $v \in$ $W_{0}^{1, p}(\Omega, w)$ and for $\eta$ small enough, we have $v \geq \psi$, thus $v$ is an admissible test function in $\left(\mathscr{P}_{n}\right)$, then

$$
\begin{aligned}
\int_{\Omega} a(x, & \left.u_{n}, \nabla u_{n}\right) \nabla\left(\eta \exp \left(G\left(u_{n}\right)\right) T_{k}\left(u_{n}^{+}-\psi^{+}\right)\right) d x \\
& +\int_{\Omega} H_{n}\left(x, u_{n}, \nabla u_{n}\right)\left(\eta \exp \left(G\left(u_{n}\right)\right) T_{k}\left(u_{n}^{+}-\psi^{+}\right)\right) d x \\
\leq & \int_{\Omega} f_{n}\left(\eta \exp \left(G\left(u_{n}\right)\right) T_{k}\left(u_{n}^{+}-\psi^{+}\right)\right) d x
\end{aligned}
$$

which implies that

$$
\begin{aligned}
\int_{\Omega} a(x, & \left.u_{n}, \nabla u_{n}\right) \nabla\left(\exp \left(G\left(u_{n}\right)\right) T_{k}\left(u_{n}^{+}-\psi^{+}\right)\right) d x \\
& \quad+\int_{\Omega} H_{n}\left(x, u_{n}, \nabla u_{n}\right) \exp \left(G\left(u_{n}\right)\right) T_{k}\left(u_{n}^{+}-\psi^{+}\right) d x \\
\leq & \int_{\Omega} f_{n} \exp \left(G\left(u_{n}\right)\right) T_{k}\left(u_{n}^{+}-\psi^{+}\right) d x .
\end{aligned}
$$

Then,

$$
\begin{aligned}
\int_{\Omega} a(x, & \left.u_{n}, \nabla u_{n}\right) \nabla u_{n} \frac{g\left(u_{n}\right)}{\alpha} \exp \left(G\left(u_{n}\right)\right) T_{k}\left(u_{n}^{+}-\psi^{+}\right) d x \\
& +\int_{\Omega} a\left(x, u_{n}, \nabla u_{n}\right) \nabla T_{k}\left(u_{n}^{+}-\psi^{+}\right) \exp \left(G\left(u_{n}\right)\right) d x \\
\leq & -\int_{\Omega} H_{n}\left(x, u_{n}, \nabla u_{n}\right) \exp \left(G\left(u_{n}\right)\right) T_{k}\left(u_{n}^{+}-\psi^{+}\right) d x \\
& +\int_{\Omega} f_{n} \exp \left(G\left(u_{n}\right)\right) T_{k}\left(u_{n}^{+}-\psi^{+}\right) d x \\
\leq & \int_{\Omega} \gamma(x) \exp \left(G\left(u_{n}\right)\right) T_{k}\left(u_{n}^{+}-\psi^{+}\right) d x \\
& +\int_{\Omega} g\left(u_{n}\right) \sum_{i=1}^{N}\left|\frac{\partial u_{n}}{\partial x_{i}}\right|^{p} w_{i} \exp \left(G\left(u_{n}\right)\right) T_{k}\left(u_{n}^{+}-\psi^{+}\right) d x \\
& +\int_{\Omega} f_{n} \exp \left(G\left(u_{n}\right)\right) T_{k}\left(u_{n}^{+}-\psi^{+}\right) d x .
\end{aligned}
$$


18 Quasilinear degenerate elliptic unilateral problems

In view of (3.9), we obtain

$$
\begin{aligned}
& \int_{\Omega} a(x,\left.u_{n}, \nabla u_{n}\right) \nabla T_{k}\left(u_{n}^{+}-\psi^{+}\right) \exp \left(G\left(u_{n}\right)\right) d x \\
& \leq \int_{\Omega} \gamma(x) \exp \left(G\left(u_{n}\right)\right) T_{k}\left(u_{n}^{+}-\psi^{+}\right) d x \\
& \quad+\int_{\Omega} f_{n} \exp \left(G\left(u_{n}\right)\right) T_{k}\left(u_{n}^{+}-\psi^{+}\right) d x \\
& \leq c_{1} k
\end{aligned}
$$

where $c_{1}$ is a positive constant and does not depend on $n$.

Consequently, we have

$$
\begin{aligned}
\int_{\left\{\left|u_{n}^{+}-\psi^{+}\right| \leq k\right\}} & a\left(x, u_{n}, \nabla u_{n}\right) \nabla u_{n}^{+} \exp \left(G\left(u_{n}\right)\right) d x \\
\leq & \int_{\left\{\left|u_{n}^{+}-\psi^{+}\right| \leq k\right\}} a\left(x, u_{n}, \nabla u_{n}\right) \nabla \psi^{+} \exp \left(G\left(u_{n}\right)\right) d x+c_{1} k
\end{aligned}
$$

Thanks to (3.9) and Young's inequality, we obtain

$$
\int_{\left\{\left|u_{n}^{+}-\psi^{+}\right| \leq k\right\}} \sum_{i=1}^{N}\left|\frac{\partial u_{n}^{+}}{\partial x_{i}}\right|^{p} w_{i} d x \leq c_{2} k .
$$

Since $\left\{x \in \Omega,\left|u_{n}^{+}\right| \leq k\right\} \subset\left\{x \in \Omega,\left|u_{n}^{+}-\psi^{+}\right| \leq k+\left\|\psi^{+}\right\|_{\infty}\right\}$, hence

$$
\begin{aligned}
& \int_{\Omega} \sum_{i=1}^{N}\left|\frac{\partial T_{k}\left(u_{n}^{+}\right)}{\partial x_{i}}\right|^{p} w_{i} d x \\
& \quad=\int_{\left\{\left|u_{n}^{+}\right| \leq k\right\}} \sum_{i=1}^{N}\left|\frac{\partial u_{n}^{+}}{\partial x_{i}}\right|^{p} w_{i} d x \leq \int_{\left\{\left|u_{n}^{+}-\psi^{+}\right| \leq k+\left\|\psi^{+}\right\|_{\infty}\right\}} \sum_{i=1}^{N}\left|\frac{\partial u_{n}^{+}}{\partial x_{i}}\right|^{p} w_{i} d x,
\end{aligned}
$$

from which in addition to (3.10) we deduce that

$$
\int_{\Omega} \sum_{i=1}^{N}\left|\frac{\partial T_{k}\left(u_{n}^{+}\right)}{\partial x_{i}}\right|^{p} w_{i} d x \leq c_{3} k \quad \forall k>0,
$$

where $c_{3}$ is a positive constant.

On the other hand, taking $v=u_{n}+\exp \left(-G\left(u_{n}\right)\right) T_{k}\left(u_{n}^{-}\right)$as the test function in $\left(\mathscr{P}_{n}\right)$, we obtain

$$
\begin{aligned}
-\int_{\Omega} a\left(x, u_{n}, \nabla u_{n}\right) \nabla\left(\eta \exp \left(-G\left(u_{n}\right)\right) T_{k} u_{n}^{-}\right) d x \\
\quad-\int_{\Omega} H_{n}\left(x, u_{n}, \nabla u_{n}\right)\left(\eta \exp \left(-G\left(u_{n}\right)\right) T_{k}\left(u_{n}^{-}\right)\right) d x \\
\leq-\int_{\Omega} f_{n}\left(\eta \exp \left(-G\left(u_{n}\right)\right) T_{k}\left(u_{n}^{-}\right)\right) d x .
\end{aligned}
$$


Using (3.10), we can write

$$
\begin{aligned}
\int_{\Omega} a( & \left.x, u_{n}, \nabla u_{n}\right) \nabla u_{n} \frac{g\left(u_{n}\right)}{\alpha} \exp \left(-G\left(u_{n}\right)\right) T_{k}\left(u_{n}^{-}\right) d x \\
& -\int_{\Omega} a\left(x, u_{n}, \nabla u_{n}\right) \nabla T_{k}\left(u_{n}^{-}\right) \exp \left(-G\left(u_{n}\right)\right) d x \\
\leq & \int_{\Omega} \gamma(x) \exp \left(-G\left(u_{n}\right)\right) T_{k}\left(u_{n}^{-}\right) d x \\
& +\int_{\Omega} g\left(u_{n}\right) \sum_{i=1}^{N}\left|\frac{\partial u_{n}}{\partial x_{i}}\right|^{p} w_{i} \exp \left(-G\left(u_{n}\right)\right) T_{k}\left(u_{n}^{-}\right) d x \\
& -\int_{\Omega} f_{n} \exp \left(-G\left(u_{n}\right)\right) T_{k}\left(u_{n}^{-}\right) d x .
\end{aligned}
$$

In virtue of (3.9) and since the functions $\gamma$ and $f_{n}$ lie in $L^{1}(\Omega)$ with $\left\|f_{n}\right\|_{L^{1}(\Omega)} \leq\|f\|_{L^{1}(\Omega)}$, we get

$$
\begin{aligned}
&-\int_{\Omega} a\left(x, u_{n}, \nabla u_{n}\right) \nabla T_{k}\left(u_{n}^{-}\right) \exp \left(-G\left(u_{n}\right)\right) d x \\
& \quad \int_{\left\{u_{n} \leq 0\right\}} a\left(x, u_{n}, \nabla u_{n}\right) \nabla T_{k}\left(u_{n}\right) \exp \left(-G\left(u_{n}\right)\right) d x \\
& \quad \leq c_{3} k .
\end{aligned}
$$

By using, again, (3.9), we deduce that

$$
\int_{\left\{u_{n} \leq 0\right\}} \sum_{i=1}^{N}\left|\frac{\partial T_{k}\left(u_{n}\right)}{\partial x_{i}}\right|^{p} w_{i} d x \leq c_{3} k
$$

that is,

$$
\int_{\Omega} \sum_{i=1}^{N}\left|\frac{\partial T_{k}\left(u_{n}^{-}\right)}{\partial x_{i}}\right|^{p} w_{i} d x \leq c_{4} k,
$$

where $c_{4}$ is a positive constant.

Combining (3.30) and (3.35), we conclude (3.22).

\subsection{Strong convergence of truncations}

Proposition 3.6. Let $u_{n}$ be solutions of the problems $\left(\mathscr{P}_{n}\right)$, then there exists a measurable function $u$ such that

$$
T_{k}\left(u_{n}\right) \longrightarrow T_{k}(u) \text { strongly in } W_{0}^{1, p}(\Omega, w) .
$$

Proof.

Step 1. We prove that $u_{n}$ converges to some function $u$ locally in measure (and therefore we can always assume that the convergence is a.e. in $\Omega$ after passing to a suitable subsequence). We will show that $u_{n}$ is a Cauchy sequence in measure in any ball $B_{R}$. 
On the one hand, thanks to (3.22), we have

$$
\begin{aligned}
& T_{k}\left(u_{n}\right) \hookrightarrow v_{k} \quad \text { weakly in } W_{0}^{1, p}(\Omega, w), \\
& T_{k}\left(u_{n}\right) \longrightarrow v_{k} \quad \text { strongly in } L^{q}(\Omega, \sigma) \text { and a.e. in } \Omega,
\end{aligned}
$$

for some function $v_{k} \in W_{0}^{1, p}(\Omega, w)$.

On the other hand, letting $k>0$ large enough, we have

$$
\begin{aligned}
k \text { meas }\left(\left\{\left|u_{n}\right|>k\right\} \cap B_{R}\right) & =\int_{\left\{\left\{\left|u_{n}\right|>k\right\} \cap B_{R}\right\}}\left|T_{k}\left(u_{n}\right)\right| d x \leq \int_{B_{R}}\left|T_{k}\left(u_{n}\right)\right| d x \\
& \leq\left(\int_{\Omega}\left|T_{k}\left(u_{n}\right)\right|^{q} \sigma d x\right)^{1 / q}\left(\int_{B_{R}} \sigma^{1-q^{\prime}} d x\right)^{1 / q^{\prime}} \\
& \leq c_{R}\left(\int_{\Omega} \sum_{i=1}^{N}\left|\frac{\partial T_{k}\left(u_{n}\right)}{\partial x_{i}}\right|^{p} w_{i}(x) d x\right)^{1 / p} \quad(\text { due to (3.5)) } \\
& \leq c k^{1 / p} \quad(\text { due to }(3.22))
\end{aligned}
$$

which implies that

$$
\operatorname{meas}\left(\left\{\left|u_{n}\right|>k\right\} \cap B_{R}\right) \leq \frac{c}{k^{1-(1 / p)}} \quad \forall k>1 \text {. }
$$

For every $\delta>0$, we have

$$
\begin{aligned}
\operatorname{meas}( & \left.\left\{\left|u_{n}-u_{m}\right|>\delta\right\} \cap B_{R}\right) \\
\leq & \text { meas }\left(\left\{\left|u_{n}\right|>k\right\} \cap B_{R}\right)+\text { meas }\left(\left\{\left|u_{m}\right|>k\right\} \cap B_{R}\right) \\
& + \text { meas }\left\{\left|T_{k}\left(u_{n}\right)-T_{k}\left(u_{m}\right)\right|>\delta\right\} .
\end{aligned}
$$

Let $\varepsilon>0$, combining (3.37), (3.39), and (3.40), we can deduce that there exists some $k(\varepsilon)>0$ such that meas $\left(\left\{\left|u_{n}-u_{m}\right|>\delta\right\} \cap B_{R}\right)<\varepsilon$ for all $n, m \geq n_{0}(k(\varepsilon), \delta, R)$. This proves that $\left(u_{n}\right)$ is a Cauchy sequence in measure in $B_{R}$, thus it converges almost everywhere to some measurable function $u$. Then by using (3.37), we have

$$
\begin{aligned}
& T_{k}\left(u_{n}\right) \hookrightarrow T_{k}(u) \quad \text { weakly in } W_{0}^{1, p}(\Omega, w), \\
& T_{k}\left(u_{n}\right) \longrightarrow T_{k}(u) \quad \text { strongly in } L^{q}(\Omega, \sigma) \text { and a.e. in } \Omega .
\end{aligned}
$$

Step 2. We claim that

$$
\lim _{j \rightarrow \infty} \lim _{n \rightarrow \infty} \int_{\left\{j \leq\left|u_{n}\right| \leq j+1\right\}} a\left(x, u_{n}, \nabla u_{n}\right) \nabla u_{n} d x=0 .
$$

Consider the function $v=u_{n}-\eta \exp \left(G\left(u_{n}\right)\right) T_{1}\left(u_{n}-T_{j}\left(u_{n}\right)\right)^{+}$. 
For $j$ large enough and $\eta$ small enough, we can deduce that $v \geq \psi$, and since $v \in$ $W_{0}^{1, p}(\Omega, w)$, hence $v$ is a test function in $\left(\mathscr{P}_{n}\right)$. Then, we obtain

$$
\begin{aligned}
& \int_{\Omega} a\left(x, u_{n}, \nabla u_{n}\right) \nabla\left(\exp \left(G\left(u_{n}\right)\right) T_{1}\left(u_{n}-T_{j}\left(u_{n}\right)\right)^{+}\right) d x \\
& \quad+\int_{\Omega} H_{n}\left(x, u_{n}, \nabla u_{n}\right) \exp \left(G\left(u_{n}\right)\right) T_{1}\left(u_{n}-T_{j}\left(u_{n}\right)\right)^{+} d x \\
& \leq \int_{\Omega} f_{n} \exp \left(G\left(u_{n}\right)\right) T_{1}\left(u_{n}-T_{j}\left(u_{n}\right)\right)^{+} d x .
\end{aligned}
$$

From the growth condition (3.10), we have

$$
\begin{aligned}
& \int_{\Omega} a\left(x, u_{n}, \nabla u_{n}\right) \nabla u_{n} \frac{g\left(u_{n}\right)}{\alpha} \exp \left(G\left(u_{n}\right)\right) T_{1}\left(u_{n}-T_{j}\left(u_{n}\right)\right)^{+} d x \\
& \quad+\int_{\Omega} a\left(x, u_{n}, \nabla u_{n}\right) \nabla T_{1}\left(u_{n}-T_{j}\left(u_{n}\right)\right)^{+} \exp \left(G\left(u_{n}\right)\right) d x \\
& \leq \int_{\Omega} \gamma(x) \exp \left(G\left(u_{n}\right)\right) T_{1}\left(u_{n}-T_{j}\left(u_{n}\right)\right)^{+} d x \\
& \quad+\int_{\Omega} g\left(u_{n}\right) \sum_{i=1}^{N}\left|\frac{\partial u_{n}}{\partial x_{i}}\right|^{p} w_{i} \exp \left(G\left(u_{n}\right)\right) T_{1}\left(u_{n}-T_{j}\left(u_{n}\right)\right)^{+} d x \\
& \quad+\int_{\Omega} f_{n} \exp \left(G\left(u_{n}\right)\right) T_{1}\left(u_{n}-T_{j}\left(u_{n}\right)\right)^{+} d x,
\end{aligned}
$$

which gives by using (3.9) the following inequality:

$$
\begin{gathered}
\int_{\Omega} a\left(x, u_{n}, \nabla u_{n}\right) \nabla T_{1}\left(u_{n}-T_{j}\left(u_{n}\right)\right)^{+} \exp \left(G\left(u_{n}\right)\right) d x \\
\leq \int_{\Omega} \gamma(x) \exp \left(G\left(u_{n}\right)\right) T_{1}\left(u_{n}-T_{j}\left(u_{n}\right)\right)^{+} d x \\
\quad+\int_{\Omega} f_{n} \exp \left(G\left(u_{n}\right)\right) T_{1}\left(u_{n}-T_{j}\left(u_{n}\right)\right)^{+} d x
\end{gathered}
$$

By Lebesgue's theorem the right-hand side goes to zero as $n$ and $j$ tend to infinity. Therefore, passing to the limit firstly in $n$ and secondly in $j$, we obtain from (3.45)

$$
\lim _{j \rightarrow \infty} \lim _{n \rightarrow \infty} \int_{\left\{j \leq u_{n} \leq j+1\right\}} a\left(x, u_{n}, \nabla u_{n}\right) \nabla u_{n} d x=0 .
$$

On the other hand, taking $v=u_{n}+\exp \left(-G\left(u_{n}\right)\right) T_{1}\left(u_{n}-T_{j}\left(u_{n}\right)\right)^{-}$in $\left(\mathscr{P}_{n}\right)$ (which is an admissible test function), we obtain

$$
\begin{aligned}
& \int_{\Omega} a\left(x, u_{n}, \nabla u_{n}\right) \nabla\left(-\exp \left(-G\left(u_{n}\right)\right) T_{1}\left(u_{n}-T_{j}\left(u_{n}\right)\right)^{-}\right) d x \\
& \quad+\int_{\Omega} H_{n}\left(x, u_{n}, \nabla u_{n}\right)\left(-\exp \left(-G\left(u_{n}\right)\right) T_{1}\left(u_{n}-T_{j}\left(u_{n}\right)\right)^{-}\right) d x \\
& \leq \int_{\Omega} f_{n}\left(-\exp \left(-G\left(u_{n}\right)\right) T_{1}\left(u_{n}-T_{j}\left(u_{n}\right)\right)^{-}\right) d x,
\end{aligned}
$$


22 Quasilinear degenerate elliptic unilateral problems

which implies that

$$
\begin{gathered}
\int_{\Omega} a\left(x, u_{n}, \nabla u_{n}\right) \nabla u_{n} \frac{g\left(u_{n}\right)}{\alpha} \exp \left(-G\left(u_{n}\right)\right) T_{1}\left(u_{n}-T_{j}\left(u_{n}\right)\right)^{-} d x \\
\quad-\int_{\Omega} a\left(x, u_{n}, \nabla u_{n}\right) \nabla T_{1}\left(u_{n}-T_{j}\left(u_{n}\right)\right)^{-} \exp \left(-G\left(u_{n}\right)\right) d x \\
\leq \int_{\Omega} H_{n}\left(x, u_{n}, \nabla u_{n}\right) \exp \left(-G\left(u_{n}\right)\right) T_{1}\left(u_{n}-T_{j}\left(u_{n}\right)\right)^{-} d x \\
\quad-\int_{\Omega} f_{n} \exp \left(-G\left(u_{n}\right)\right) T_{1}\left(u_{n}-T_{j}\left(u_{n}\right)\right)^{-} d x .
\end{gathered}
$$

In virtue of (3.9) and (3.10), it is possible to conclude that

$$
\begin{gathered}
-\int_{\Omega} a\left(x, u_{n}, \nabla u_{n}\right) \nabla T_{1}\left(u_{n}-T_{j}\left(u_{n}\right)\right)^{-} \exp \left(-G\left(u_{n}\right)\right) d x \\
\leq \int_{\Omega} \gamma(x) \exp \left(-G\left(u_{n}\right)\right) T_{1}\left(u_{n}-T_{j}\left(u_{n}\right)\right)^{-} d x \\
\quad-\int_{\Omega} f_{n} \exp \left(-G\left(u_{n}\right)\right) T_{1}\left(u_{n}-T_{j}\left(u_{n}\right)\right)^{-} d x .
\end{gathered}
$$

The second term in the right-hand side of the previous inequality can be neglected since it is nonnegative, and by Lebesgue's theorem the first term goes to zero as $n$ and $j$ tend to infinity. Then (3.49) becomes

$$
\lim _{j \rightarrow \infty} \lim _{n \rightarrow \infty} \int_{\left\{-j-1 \leq u_{n} \leq-j\right\}} a\left(x, u_{n}, \nabla u_{n}\right) \nabla u_{n} d x=0 .
$$

Finally, (3.42) follows from (3.46) and (3.50).

Step 3. We will show that

$$
\lim _{j \rightarrow \infty} \lim _{n \rightarrow \infty} \int_{\Omega} a\left(x, T_{k}\left(u_{n}\right), \nabla T_{k}\left(u_{n}\right)\right) \nabla T_{k}\left(u_{n}\right)\left(1-h_{j}\left(u_{n}\right)\right) d x=0,
$$

where $h_{j}$ is a real variable function defined as

$$
\begin{aligned}
& h_{j}(s)=1 \quad \text { if }|s| \leq j \\
& h_{j}(s)=0 \quad \text { if }|s| \geq j+1, \\
& h_{j}(s)=j+1-s \quad \text { if } j \leq s \leq j+1, \\
& h_{j}(s)=s+j+1 \quad \text { if }-j-1 \leq s \leq-j,
\end{aligned}
$$

with $j$ a nonnegative real parameter. 
Let $v=u_{n}+\exp \left(-G\left(u_{n}\right)\right) T_{k}\left(u_{n}\right)^{-}\left(1-h_{j}\left(u_{n}\right)\right), v$ a test function in $\left(\mathscr{P}_{n}\right)$. Then we have

$$
\begin{aligned}
& \int_{\Omega} a\left(x, u_{n}, \nabla u_{n}\right) \nabla\left(-\exp \left(-G\left(u_{n}\right)\right) T_{k}\left(u_{n}\right)^{-}\left(1-h_{j}\left(u_{n}\right)\right)\right) d x \\
& \quad+\int_{\Omega} H_{n}\left(x, u_{n}, \nabla u_{n}\right)\left(-\exp \left(-G\left(u_{n}\right)\right) T_{k}\left(u_{n}\right)^{-}\left(1-h_{j}\left(u_{n}\right)\right)\right) d x \\
& \leq \int_{\Omega} f_{n}\left(-\exp \left(-G\left(u_{n}\right)\right) T_{k}\left(u_{n}\right)^{-}\left(1-h_{j}\left(u_{n}\right)\right)\right) d x .
\end{aligned}
$$

By using (3.10), we have

$$
\begin{aligned}
\int_{\Omega} a( & \left.x, u_{n}, \nabla u_{n}\right) \nabla u_{n} \frac{g\left(u_{n}\right)}{\alpha} \exp \left(-G\left(u_{n}\right)\right) T_{k}\left(u_{n}\right)^{-}\left(1-h_{j}\left(u_{n}\right)\right) d x \\
& -\int_{\Omega} a\left(x, u_{n}, \nabla u_{n}\right) \nabla T_{k}\left(u_{n}\right)^{-} \exp \left(-G\left(u_{n}\right)\right)\left(1-h_{j}\left(u_{n}\right)\right) d x \\
& +\int_{\Omega} a\left(x, u_{n}, \nabla u_{n}\right) \nabla h_{j}\left(u_{n}\right) \exp \left(-G\left(u_{n}\right)\right) T_{k}\left(u_{n}\right)^{-} d x \\
& -\int_{\Omega} \gamma(x) \exp \left(-G\left(u_{n}\right)\right) T_{k}\left(u_{n}\right)^{-}\left(1-h_{j}\left(u_{n}\right)\right) d x \\
& -\int_{\Omega} \exp \left(-G\left(u_{n}\right)\right) g\left(u_{n}\right) \sum_{i=1}^{N}\left|\frac{\partial u_{n}}{\partial x_{i}}\right|^{p} w_{i} T_{k}\left(u_{n}\right)^{-}\left(1-h_{j}\left(u_{n}\right)\right) d x \\
\leq & -\int_{\Omega} f_{n} \exp \left(-G\left(u_{n}\right)\right) T_{k}\left(u_{n}\right)^{-}\left(1-h_{j}\left(u_{n}\right)\right) d x .
\end{aligned}
$$

Thanks to (3.9), we can deduce that

$$
\begin{aligned}
& -\int_{\left\{u_{n} \leq 0\right\}} a\left(x, u_{n}, \nabla u_{n}\right) \nabla T_{k}\left(u_{n}\right) \exp \left(-G\left(u_{n}\right)\right)\left(1-h_{j}\left(u_{n}\right)\right) d x \\
& \quad-\int_{\left\{-j-1 \leq u_{n} \leq-j\right\}} a\left(x, u_{n}, \nabla u_{n}\right) \nabla u_{n} \exp \left(-G\left(u_{n}\right)\right) T_{k}\left(u_{n}\right)^{-} d x \\
& \quad+\int_{\Omega} \gamma(x) \exp \left(-G\left(u_{n}\right)\right) T_{k}\left(u_{n}\right)^{-}\left(1-h_{j}\left(u_{n}\right)\right) d x \\
& \geq \int_{\Omega} f_{n} \exp \left(-G\left(u_{n}\right)\right) T_{k}\left(u_{n}\right)^{-}\left(1-h_{j}\left(u_{n}\right)\right) d x .
\end{aligned}
$$

In view of (3.35), the second integral tends to zero as $n$ and $j$ go to infinity. And by Lebesgue's theorem, it is possible to conclude that the third and fourth integrals converge to zero as $n$ and $j$ go to infinity. Then (3.41) implies that

$$
\lim _{j \rightarrow \infty} \lim _{n \rightarrow \infty} \int_{\left\{u_{n} \leq 0\right\}} a\left(x, T_{k}\left(u_{n}\right), \nabla T_{k}\left(u_{n}\right)\right) \nabla T_{k}\left(u_{n}\right)\left(1-h_{j}\left(u_{n}\right)\right) d x=0 .
$$


On the other hand, take $v=u_{n}-\eta \exp \left(G\left(u_{n}\right)\right) T_{k}\left(u_{n}^{+}-\psi^{+}\right)\left(1-h_{j}\left(u_{n}\right)\right)$ which is an admissible test function in $\left(\mathscr{P}_{n}\right)$, then, we have

$$
\begin{aligned}
& \int_{\Omega} a\left(x, u_{n}, \nabla u_{n}\right) \nabla\left(\eta \exp \left(G\left(u_{n}\right)\right) T_{k}\left(u_{n}^{+}-\psi^{+}\right)\left(1-h_{j}\left(u_{n}\right)\right)\right) d x \\
& \quad+\int_{\Omega} H_{n}\left(x, u_{n}, \nabla u_{n}\right)\left(\eta \exp \left(G\left(u_{n}\right)\right) T_{k}\left(u_{n}^{+}-\psi^{+}\right)\left(1-h_{j}\left(u_{n}\right)\right)\right) d x \\
& \leq \int_{\Omega} f_{n}\left(\eta \exp \left(G\left(u_{n}\right)\right) T_{k}\left(u_{n}^{+}-\psi^{+}\right)\left(1-h_{j}\left(u_{n}\right)\right)\right) d x,
\end{aligned}
$$

which in addition to (3.10) implies that

$$
\begin{aligned}
& \int_{\Omega} a\left(x, u_{n}, \nabla u_{n}\right) \nabla u_{n} \frac{g\left(u_{n}\right)}{\alpha} \exp \left(G\left(u_{n}\right)\right) T_{k}\left(u_{n}^{+}-\psi^{+}\right)\left(1-h_{j}\left(u_{n}\right)\right) d x \\
& \quad+\int_{\Omega} a\left(x, u_{n}, \nabla u_{n}\right) \nabla T_{k}\left(u_{n}^{+}-\psi^{+}\right) \exp \left(G\left(u_{n}\right)\right)\left(1-h_{j}\left(u_{n}\right)\right) d x \\
& \quad+\int_{\left\{j \leq u_{n} \leq j+1\right\}} a\left(x, u_{n}, \nabla u_{n}\right) \nabla u_{n} \exp \left(G\left(u_{n}\right)\right) T_{k}\left(u_{n}^{+}-\psi^{+}\right) d x \\
& \leq \int_{\Omega} g\left(u_{n}\right) \sum_{i=1}^{N}\left|\frac{\partial u_{n}}{\partial x_{i}}\right|^{p} w_{i} \exp \left(G\left(u_{n}\right)\right) T_{k}\left(u_{n}^{+}-\psi^{+}\right)\left(1-h_{j}\left(u_{n}\right)\right) d x \\
& \quad+\int_{\Omega} f_{n} \exp \left(G\left(u_{n}\right)\right) T_{k}\left(u_{n}^{+}-\psi^{+}\right)\left(1-h_{j}\left(u_{n}\right)\right) d x \\
& \quad+\int_{\Omega} \gamma(x) \exp \left(G\left(u_{n}\right)\right) T_{k}\left(u_{n}^{+}-\psi^{+}\right)\left(1-h_{j}\left(u_{n}\right)\right) d x,
\end{aligned}
$$

which takes, by using (3.9), the form

$$
\begin{aligned}
& \int_{\Omega} a\left(x, u_{n}, \nabla u_{n}\right) \nabla T_{k}\left(u_{n}^{+}-\psi^{+}\right) \exp \left(G\left(u_{n}\right)\right)\left(1-h_{j}\left(u_{n}\right)\right) d x \\
& \leq- \int_{\left\{j \leq u_{n} \leq j+1\right\}} a\left(x, u_{n}, \nabla u_{n}\right) \nabla u_{n} \exp \left(G\left(u_{n}\right)\right) T_{k}\left(u_{n}^{+}-\psi^{+}\right) d x \\
&+\int_{\Omega} \gamma(x) \exp \left(G\left(u_{n}\right)\right) T_{k}\left(u_{n}^{+}-\psi^{+}\right)\left(1-h_{j}\left(u_{n}\right)\right) d x \\
&+\int_{\Omega} f_{n} \exp \left(G\left(u_{n}\right)\right) T_{k}\left(u_{n}^{+}-\psi^{+}\right)\left(1-h_{j}\left(u_{n}\right)\right) d x \\
&= \varepsilon_{1}(j, n) .
\end{aligned}
$$

In virtue of (3.42) and Lebesgue's theorem, we can conclude that $\varepsilon_{1}(j, n)$ converges to zero as $n$ and $j$ go to infinity.

From (3.59), we have

$$
\begin{aligned}
& \int_{\left\{\left|u_{n}^{+}-\psi^{+}\right| \leq k\right\}} a\left(x, u_{n}, \nabla u_{n}\right) \nabla u_{n}^{+} \exp \left(G\left(u_{n}\right)\right)\left(1-h_{j}\left(u_{n}\right)\right) d x \\
& \quad \leq \int_{\left\{\left|u_{n}^{+}-\psi^{+}\right| \leq k\right\}} a\left(x, u_{n}, \nabla u_{n}\right) \nabla \psi^{+} \exp \left(G\left(u_{n}\right)\right)\left(1-h_{j}\left(u_{n}\right)\right) d x+\varepsilon_{1}(j, n) .
\end{aligned}
$$


Thanks to the growth condition (3.7) and Young's inequality, it is possible to conclude that

$$
\int_{\left\{\left|u_{n}^{+}-\psi^{+}\right| \leq k\right\}} a\left(x, u_{n}, \nabla u_{n}\right) \nabla u_{n}^{+} \exp \left(G\left(u_{n}\right)\right)\left(1-h_{j}\left(u_{n}\right)\right) d x \leq \varepsilon_{2}(j, n),
$$

where $\varepsilon_{2}(j, n)$ tends to 0 as $n$ and $j$ go to infinity.

Since $\exp \left(G\left(u_{n}\right)\right)$ is bounded, then

$$
\int_{\left\{\left|u_{n}^{+}-\psi^{+}\right| \leq k\right\}} a\left(x, u_{n}, \nabla u_{n}\right) \nabla u_{n}^{+} \exp \left(G\left(u_{n}\right)\right)\left(1-h_{j}\left(u_{n}\right)\right) d x \leq \varepsilon_{3}(j, n)
$$

Since $\left\{x \in \Omega,\left|u_{n}^{+}\right| \leq h\right\} \subset\left\{x \in \Omega,\left|u_{n}^{+}-\psi^{+}\right| \leq h+\left\|\psi^{+}\right\|_{\infty}\right\}$, hence

$$
\begin{aligned}
\int_{\left\{\left|u_{n}^{+}\right| \leq k\right\}} a\left(x, u_{n}, \nabla u_{n}\right) \nabla u_{n}\left(1-h_{j}\left(u_{n}\right)\right) d x \\
\quad \leq \int_{\left\{\left|u_{n}^{+}-\psi^{+}\right| \leq k+\left\|\psi^{+}\right\|_{\infty}\right\}} a\left(x, u_{n}, \nabla u_{n}\right) \nabla u_{n}\left(1-h_{j}\left(u_{n}\right)\right) d x \\
\quad \leq \varepsilon_{3}(j, n),
\end{aligned}
$$

which yields, for all $k>0$,

$$
\lim _{j \rightarrow \infty} \lim _{n \rightarrow \infty} \int_{\left\{u_{n} \geq 0\right\}} a\left(x, T_{k}\left(u_{n}\right), \nabla T_{k}\left(u_{n}\right)\right) \nabla T_{k}\left(u_{n}\right)\left(1-h_{j}\left(u_{n}\right)\right) d x=0
$$

Using (3.56) and (3.64), we conclude (3.51).

Step 4. We prove that

$$
\begin{gathered}
\lim _{j \rightarrow \infty} \lim _{n \rightarrow \infty} \int_{\Omega}\left(a\left(x, T_{k}\left(u_{n}\right), \nabla T_{k}\left(u_{n}\right)\right)-a\left(x, T_{k}\left(u_{n}\right), \nabla T_{k}(u)\right)\right) \\
\times\left(\nabla T_{k}\left(u_{n}\right)-\nabla T_{k}(u)\right) h_{j}\left(u_{n}\right) d x=0 .
\end{gathered}
$$

On the one hand, let $v=u_{n}-\eta \exp \left(G\left(u_{n}\right)\right)\left(T_{k}\left(u_{n}\right)-T_{k}(u)\right)^{+} h_{j}\left(u_{n}\right)$ with $h_{j}$ defined as in (3.52) and $\eta$ small enough such that $v \in K_{\psi}$, then, we take $v$ as the test function in $\left(\mathscr{P}_{n}\right)$ and we obtain

$$
\begin{aligned}
& \int_{\Omega} a\left(x, u_{n}, \nabla u_{n}\right) \nabla\left(\eta \exp \left(G\left(u_{n}\right)\right)\left(T_{k}\left(u_{n}\right)-T_{k}(u)\right)^{+} h_{j}\left(u_{n}\right)\right) d x \\
& \quad+\int_{\Omega} H_{n}\left(x, u_{n}, \nabla u_{n}\right)\left(\eta \exp \left(G\left(u_{n}\right)\right)\left(T_{k}\left(u_{n}\right)-T_{k}(u)\right)^{+} h_{j}\left(u_{n}\right)\right) d x \\
& \leq \int_{\Omega} f_{n}\left(\eta \exp \left(G\left(u_{n}\right)\right)\left(T_{k}\left(u_{n}\right)-T_{k}(u)\right)^{+} h_{j}\left(u_{n}\right)\right) d x .
\end{aligned}
$$


Similarly, using (3.9) and (3.10), we obtain

$$
\begin{aligned}
& \int_{\Omega} a\left(x, u_{n}, \nabla u_{n}\right) \nabla\left(T_{k}\left(u_{n}\right)-T_{k}(u)\right)^{+} \exp \left(G\left(u_{n}\right)\right) h_{j}\left(u_{n}\right) d x \\
& \quad-\int_{\left\{j \leq u_{n} \leq j+1\right\}} a\left(x, u_{n}, \nabla u_{n}\right) \nabla u_{n} \exp \left(G\left(u_{n}\right)\right)\left(T_{k}\left(u_{n}\right)-T_{k}(u)\right)^{+} d x \\
& \leq \int_{\Omega} \gamma(x) \exp \left(G\left(u_{n}\right)\right)\left(T_{k}\left(u_{n}\right)-T_{k}(u)\right)^{+} h_{j}\left(u_{n}\right) d x \\
& \quad+\int_{\Omega} f_{n} \exp \left(G\left(u_{n}\right)\right)\left(T_{k}\left(u_{n}\right)-T_{k}(u)\right)^{+} h_{j}\left(u_{n}\right) d x
\end{aligned}
$$

that is,

$$
\int_{\left\{T_{k}\left(u_{n}\right)-T_{k}(u) \geq 0\right\}} a\left(x, u_{n}, \nabla u_{n}\right) \nabla\left(T_{k}\left(u_{n}\right)-T_{k}(u)\right) \exp \left(G\left(u_{n}\right)\right) h_{j}\left(u_{n}\right) d x \leq \varepsilon_{4}(j, n) .
$$

Applying again (3.42) and Lebesgue's theorem, we deduce that $\varepsilon_{4}(j, n)$ goes to zero as $n$ and $j$ tend to infinity. Moreover, (3.68) becomes

$$
\begin{aligned}
& \int_{\left\{T_{k}\left(u_{n}\right)-T_{k}(u) \geq 0\right\}} a\left(x, T_{k}\left(u_{n}\right), \nabla T_{k}\left(u_{n}\right)\right) \nabla\left(T_{k}\left(u_{n}\right)-T_{k}(u)\right) \exp \left(G\left(u_{n}\right)\right) h_{j}\left(u_{n}\right) d x \\
& \quad+\int_{\left\{T_{k}\left(u_{n}\right)-T_{k}(u) \geq 0,\left|u_{n}\right|>k\right\}} a\left(x, u_{n}, \nabla u_{n}\right) \nabla T_{k}(u) \exp \left(G\left(u_{n}\right)\right) h_{j}\left(u_{n}\right) d x \\
& \quad \leq \varepsilon_{4}(j, n) .
\end{aligned}
$$

Since $h_{j}\left(u_{n}\right)=0$ if $\left|u_{n}\right|>j+1$, hence, we obtain

$$
\begin{aligned}
& \int_{\left\{T_{k}\left(u_{n}\right)-T_{k}(u) \geq 0,\left|u_{n}\right|>k\right\}} a\left(x, u_{n}, \nabla u_{n}\right) \nabla T_{k}(u) \exp \left(G\left(u_{n}\right)\right) h_{j}\left(u_{n}\right) d x \\
& =\int_{\left\{T_{k}\left(u_{n}\right)-T_{k}(u) \geq 0,\left|u_{n}\right|>k\right\}} a\left(x, T_{j+1}\left(u_{n}\right), \nabla T_{j+1}\left(u_{n}\right)\right) \nabla T_{k}(u) \\
& \quad \times \exp \left(G\left(u_{n}\right)\right) h_{j}\left(u_{n}\right) d x \\
& \leq \varepsilon_{5}(j, n)
\end{aligned}
$$

which gives

$$
\begin{gathered}
\int_{\left\{T_{k}\left(u_{n}\right)-T_{k}(u) \geq 0\right\}} a\left(x, T_{k}\left(u_{n}\right), \nabla T_{k}\left(u_{n}\right)\right) \nabla\left(T_{k}\left(u_{n}\right)-T_{k}(u)\right) \\
\times \exp \left(G\left(u_{n}\right)\right) h_{j}\left(u_{n}\right) d x \\
\quad \leq \varepsilon_{6}(j, n),
\end{gathered}
$$

where $\varepsilon_{6}(j, n)=c\left(\int_{\left\{\left|u_{n}\right|>k\right\}}\left|a\left(x, T_{j+1}\left(u_{n}\right), \nabla T_{j+1}\left(u_{n}\right)\right)\right|\left|\nabla T_{k}(u)\right| \exp \left(G\left(u_{n}\right)\right) h_{j}\left(u_{n}\right) d x+\varepsilon_{5}(j\right.$, $n)$ ) which goes to zero as $n$ and $j$ tend to infinity. 
On the other hand, take $v=u_{n}+\exp \left(-G\left(u_{n}\right)\right)\left(T_{k}\left(u_{n}\right)-T_{k}(u)\right)^{-} h_{j}\left(u_{n}\right)$ as the test function in $\left(\mathscr{P}_{n}\right)$ and, reasoning as in (3.71), it is possible to conclude that

$$
\begin{gathered}
\lim _{j \rightarrow \infty} \lim _{n \rightarrow \infty} \int_{\left\{T_{k}\left(u_{n}\right)-T_{k}(u) \leq 0\right\}}\left(a\left(x, T_{k}\left(u_{n}\right), \nabla T_{k}\left(u_{n}\right)\right)-a\left(x, T_{k}\left(u_{n}\right), \nabla T_{k}(u)\right)\right) \\
\times\left(\nabla T_{k}\left(u_{n}\right)-\nabla T_{k}(u)\right) h_{j}\left(u_{n}\right) d x=0 .
\end{gathered}
$$

Combining (3.71) and (3.72), we deduce (3.65).

Step 5. We show that

$$
T_{k}\left(u_{n}\right) \longrightarrow T_{k}(u) \quad \text { strongly in } W_{0}^{1, p}(\Omega, w)
$$

Firstly, we have

$$
\begin{aligned}
\int_{\Omega}\left(a\left(x, T_{k}\left(u_{n}\right), \nabla T_{k}\left(u_{n}\right)\right)-a\left(x, T_{k}\left(u_{n}\right), \nabla T_{k}(u)\right)\right)\left(\nabla T_{k}\left(u_{n}\right)-\nabla T_{k}(u)\right) d x \\
=\int_{\Omega}\left(a\left(x, T_{k}\left(u_{n}\right), \nabla T_{k}\left(u_{n}\right)\right)-a\left(x, T_{k}\left(u_{n}\right), \nabla T_{k}(u)\right)\right) \\
\quad \times\left(\nabla T_{k}\left(u_{n}\right)-\nabla T_{k}(u)\right) h_{j}\left(u_{n}\right) d x \\
+\int_{\Omega}\left(a\left(x, T_{k}\left(u_{n}\right), \nabla T_{k}\left(u_{n}\right)\right)-a\left(x, T_{k}\left(u_{n}\right), \nabla T_{k}(u)\right)\right) \\
\quad \times\left(\nabla T_{k}\left(u_{n}\right)-\nabla T_{k}(u)\right)\left(1-h_{j}\left(u_{n}\right)\right) d x .
\end{aligned}
$$

Thanks to (3.65) the first integral of the right-hand side converges to zero as $n$ and $j$ tend to infinity.

For the second term, we have

$$
\begin{aligned}
\int_{\Omega}\left(a\left(x, T_{k}\left(u_{n}\right), \nabla T_{k}\left(u_{n}\right)\right)-a\left(x, T_{k}\left(u_{n}\right), \nabla T_{k}(u)\right)\right)\left(\nabla T_{k}\left(u_{n}\right)-\nabla T_{k}(u)\right)\left(1-h_{j}\left(u_{n}\right)\right) d x \\
=\int_{\Omega} a\left(x, T_{k}\left(u_{n}\right), \nabla T_{k}\left(u_{n}\right)\right) \nabla T_{k}\left(u_{n}\right)\left(1-h_{j}\left(u_{n}\right)\right) d x \\
\quad-\int_{\Omega} a\left(x, T_{k}\left(u_{n}\right), \nabla T_{k}\left(u_{n}\right)\right) \nabla T_{k}(u)\left(1-h_{j}\left(u_{n}\right)\right) d x \\
\quad-\int_{\Omega} a\left(x, T_{k}\left(u_{n}\right), \nabla T_{k}(u)\right)\left(\nabla T_{k}\left(u_{n}\right)-\nabla T_{k}(u)\right)\left(1-h_{j}\left(u_{n}\right)\right) d x .
\end{aligned}
$$

By (3.51) the first integral of the right-hand side goes to zero as $n$ and $j$ tend to infinity, and since $\left(a\left(x, T_{k}\left(u_{n}\right), \nabla T_{k}\left(u_{n}\right)\right)\right)$ is bounded in $\prod_{i=1}^{N} L^{p^{\prime}}\left(\Omega, w_{i}^{*}\right)$ uniformly on $n$ while $\nabla T_{k}(u)\left(1-h_{j}\left(u_{n}\right)\right)$ converges to zero, hence, the second integral converges to zero. For the third integral, it converges to zero because $\nabla T_{k}\left(u_{n}\right) \rightarrow \nabla T_{k}(u)$ weakly in $\prod_{i=1}^{N} L^{p}\left(\Omega, w_{i}\right)$.

Finally, we conclude that

$$
\begin{aligned}
\lim _{n \rightarrow+\infty} \int_{\Omega}\left(a\left(x, T_{k}\left(u_{n}\right), \nabla T_{k}\left(u_{n}\right)\right)-a\left(x, T_{k}\left(u_{n}\right), \nabla T_{k}(u)\right)\right) \\
\times\left(\nabla T_{k}\left(u_{n}\right)-\nabla T_{k}(u)\right) d x=0 .
\end{aligned}
$$


28 Quasilinear degenerate elliptic unilateral problems

Then [1, Lemma 3.1] implies that

$$
T_{k}\left(u_{n}\right) \longrightarrow T_{k}(u) \quad \text { strongly in } W_{0}^{1, p}(\Omega, w)
$$

This completes the proof of Proposition 3.6.

\subsection{Proof of Theorem 3.1}

Let $\varphi \in W_{0}^{1, p}(\Omega, w) \cap L^{\infty}(\Omega)$ and taking $v=T_{k}\left(u_{n}-\varphi\right)$ as test function in $\left(\mathscr{P}_{n}\right)$, we have

$$
\begin{aligned}
\int_{\Omega} a\left(x, u_{n}, \nabla u_{n}\right) \nabla T_{k}\left(u_{n}-\varphi\right) d x \\
\quad+\int_{\Omega} H_{n}\left(x, u_{n}, \nabla u_{n}\right) T_{k}\left(u_{n}-\varphi\right) d x \\
\quad \leq \int_{\Omega} f_{n} T_{k}\left(u_{n}-\varphi\right) d x,
\end{aligned}
$$

which implies that

$$
\begin{aligned}
\int_{\Omega} a & \left(x, T_{k+\|\varphi\|_{\infty}}\left(u_{n}\right), \nabla T_{k+\|\varphi\|_{\infty}}\left(u_{n}\right)\right) \nabla T_{k}\left(u_{n}-\varphi\right) d x \\
& +\int_{\Omega} H_{n}\left(x, u_{n}, \nabla u_{n}\right) T_{k}\left(u_{n}-\varphi\right) d x \\
\leq & \int_{\Omega} f_{n} T_{k}\left(u_{n}-\varphi\right) d x .
\end{aligned}
$$

In view of Proposition 3.6 and the growth condition (3.7), we deduce that

$$
\begin{aligned}
& a\left(x, T_{k+\|\varphi\|_{\infty}}\left(u_{n}\right), \nabla T_{k+\|\varphi\|_{\infty}}\left(u_{n}\right)\right) \\
& \quad \longrightarrow a\left(x, T_{k+\|\varphi\|_{\infty}}(u), \nabla T_{k+\|\varphi\|_{\infty}}(u)\right) \text { strongly in } \prod_{i=1}^{N} L^{p^{\prime}}\left(\Omega, w_{i}\right) .
\end{aligned}
$$

Moreover, using, again, Proposition 3.6, we have

$$
T_{k}\left(u_{n}-\varphi\right) \longrightarrow T_{k}(u-\varphi) \quad \text { strongly in } W_{0}^{1, p}(\Omega, w) .
$$

Then,

$$
\begin{array}{r}
\lim _{n \rightarrow \infty} \int_{\Omega} a\left(x, T_{k+\|\varphi\|_{\infty}}\left(u_{n}\right), \nabla T_{k+\|\varphi\|_{\infty}}\left(u_{n}\right)\right) T_{k}\left(u_{n}-\varphi\right) d x \\
=\int_{\Omega} a\left(x, T_{k+\|\varphi\|_{\infty}}(u), \nabla T_{k+\|\varphi\|_{\infty}}(u)\right) T_{k}(u-\varphi) d x .
\end{array}
$$

On the other hand, we claim that

$$
H_{n}\left(x, u_{n}, \nabla u_{n}\right) \longrightarrow H(x, u, \nabla u) \quad \text { strongly in } L^{1}(\Omega) \text {. }
$$


Let $v=u_{n}+\exp \left(-G\left(u_{n}\right)\right) \int_{u_{n}}^{0} g(s) \chi_{\{s<-h\}} d s$. Since $v \in W_{0}^{1, p}(\Omega, w)$ and $v \geq \psi$, hence $v$ is an admissible test function in $\left(\mathscr{P}_{n}\right)$. Then,

$$
\begin{aligned}
& \int_{\Omega} a\left(x, u_{n}, \nabla u_{n}\right) \nabla\left(-\exp \left(-G\left(u_{n}\right)\right) \int_{u_{n}}^{0} g(s) \chi_{\{s<-h\}} d s\right) d x \\
& \quad+\int_{\Omega} H_{n}\left(x, u_{n}, \nabla u_{n}\right)\left(-\exp \left(-G\left(u_{n}\right)\right) \int_{u_{n}}^{0} g(s) \chi_{\{s<-h\}} d s\right) d x \\
& \quad \leq \int_{\Omega} f_{n}\left(-\exp \left(-G\left(u_{n}\right)\right) \int_{u_{n}}^{0} g(s) \chi_{\{s<-h\}} d s\right) d x
\end{aligned}
$$

which implies that

$$
\begin{aligned}
\int_{\Omega} a( & \left.x, u_{n}, \nabla u_{n}\right) \nabla u_{n} \frac{g\left(u_{n}\right)}{\alpha} \exp \left(-G\left(u_{n}\right)\right) \int_{u_{n}}^{0} g(s) \chi_{\{s<-h\}} d s d x \\
& +\int_{\Omega} a\left(x, u_{n}, \nabla u_{n}\right) \nabla u_{n} \exp \left(-G\left(u_{n}\right)\right) g\left(u_{n}\right) \chi_{\left\{u_{n}<-h\right\}} d x \\
\leq & \int_{\Omega} \gamma(x) \exp \left(-G\left(u_{n}\right)\right) \int_{u_{n}}^{0} g(s) \chi_{\{s<-h\}} d s d x \\
& +\int_{\Omega} g\left(u_{n}\right) \sum_{i=1}^{N}\left|\frac{\partial u_{n}}{\partial x_{i}}\right|^{p} w_{i} \exp \left(-G\left(u_{n}\right)\right) \int_{u_{n}}^{0} g(s) \chi_{\{s<-h\}} d s d x \\
& -\int_{\Omega} f_{n} \exp \left(-G\left(u_{n}\right)\right) \int_{u_{n}}^{0} g(s) \chi_{\{s<-h\}} d s d x .
\end{aligned}
$$

By (3.9) and since $\int_{u_{n}}^{0} g(s) \chi_{\{s<-h\}} d s \leq \int_{-\infty}^{-h} g(s) d s$, we have

$$
\begin{array}{r}
\int_{\Omega} a\left(x, u_{n}, \nabla u_{n}\right) \nabla u_{n} \exp \left(-G\left(u_{n}\right)\right) g\left(u_{n}\right) \chi_{\left\{u_{n}<-h\right\}} d x \\
\quad \leq \exp \left(\frac{\|g\|_{L^{1}(\mathbb{R})}}{\alpha}\right) \int_{-\infty}^{-h} g(s) d s\left(\|\gamma\|_{L^{1}(\Omega)}+\left\|f_{n}\right\|_{L^{1}(\Omega)}\right) \\
\quad \leq \exp \left(\frac{\|g\|_{L^{1}(\mathbb{R})}}{\alpha}\right) \int_{-\infty}^{-h} g(s) d s\left(\|\gamma\|_{L^{1}(\Omega)}+\|f\|_{L^{1}(\Omega)}\right) .
\end{array}
$$

Using again (3.9), we obtain

$$
\int_{\left\{u_{n}<-h\right\}} g\left(u_{n}\right) \sum_{i=1}^{N}\left|\frac{\partial u_{n}}{\partial x_{i}}\right|^{p} w_{i} d x \leq c \int_{-\infty}^{-h} g(s) d s
$$

and since $g \in L^{1}(\mathbb{R})$, we deduce that

$$
\lim _{h \rightarrow+\infty} \sup _{n \in \mathbb{N}} \int_{\left\{u_{n}<-h\right\}} g\left(u_{n}\right) \sum_{i=1}^{N}\left|\frac{\partial u_{n}}{\partial x_{i}}\right|^{p} w_{i} d x=0 .
$$


On the other hand, letting $M=\exp \left(-G\left(u_{n}\right)\right) \int_{0}^{+\infty} g(s) d s$ and $h \geq M+\left\|\psi^{+}\right\|_{L^{\infty}(\Omega)}$, we consider $v=u_{n}-\exp \left(G\left(u_{n}\right)\right) \int_{0}^{u_{n}} g(s) \chi_{\{s>h\}} d s$. Since $v \in W_{0}^{1, p}(\Omega, w)$ and $v \geq \psi, v$ is an admissible test function in $\left(\mathscr{P}_{n}\right)$. Then, similarly as in (3.88), we deduce that

$$
\lim _{h \rightarrow+\infty} \sup _{n \in \mathbb{N}} \int_{\left\{u_{n}>h\right\}} g\left(u_{n}\right) \sum_{i=1}^{N}\left|\frac{\partial u_{n}}{\partial x_{i}}\right|^{p} w_{i} d x=0 .
$$

Combining (3.88), (3.89), Proposition 3.6, and Vitali's theorem, we conclude (3.60).

On the other hand, letting $\varphi \in K_{\psi} \cap L^{\infty}(\Omega)$ and taking $v=u_{n}-T_{k}\left(u_{n}-\varphi\right)$ as test function in $\left(\mathscr{P}_{n}\right)$, we get

$$
\begin{gathered}
u_{n} \in K_{\psi} \quad \forall k>0, \\
\int_{\Omega} a\left(x, u_{n}, \nabla u_{n}\right) \nabla T_{k}\left(u_{n}-\varphi\right) d x+\int_{\Omega} H_{n}\left(x, u_{n}, \nabla u_{n}\right) T_{k}\left(u_{n}-\varphi\right) d x \\
\leq \int_{\Omega} f_{n} T_{k}\left(u_{n}-\varphi\right) d x \quad \forall \varphi \in K_{\psi} \cap L^{\infty}(\Omega),
\end{gathered}
$$

which implies that

$$
\begin{gathered}
u_{n} \in K_{\psi} \quad \forall k>0, \\
\int_{\Omega} a\left(x, T_{k+\|\varphi\|_{\infty}}\left(u_{n}\right), \nabla T_{k+\|\varphi\|_{\infty}}\left(u_{n}\right)\right) \nabla T_{k}\left(u_{n}-\varphi\right) d x+\int_{\Omega} H_{n}\left(x, u_{n}, \nabla u_{n}\right) T_{k}\left(u_{n}-\varphi\right) d x \\
\leq \int_{\Omega} f_{n} T_{k}\left(u_{n}-\varphi\right) d x \quad \forall \varphi \in K_{\psi} \cap L^{\infty}(\Omega) .
\end{gathered}
$$

Finally, from (3.82) and (3.83), we can pass to the limit in (3.91). This completes the proof of Theorem 3.1 .

\section{References}

[1] Y. Akdim, E. Azroul, and A. Benkirane, Existence of solutions for quasilinear degenerate elliptic equations, Electron. J. Differential Equations 2001 (2001), no. 71, 1-19.

[2] - Strongly nonlinear degenerated elliptic unilateral problems via convergence of truncations, Proceedings of the $2002 \mathrm{Fez}$ Conference on Partial Differential Equations (Tex, 2002), Electron. J. Differ. Equ. Conf., vol. 9, Southwest Texas State University, 2002, pp. 25-39.

[3] __ Existence of solution for quasilinear degenerated elliptic unilateral problems, Ann. Math. Blaise Pascal 10 (2003), no. 1, 1-20.

[4] Existence results for quasilinear degenerated equations via strong convergence of truncations, Rev. Mat. Complut. 17 (2004), no. 2, 359-379.

[5] L. Boccardo, F. Murat, and J.-P. Puel, $L^{\infty}$ estimate for some nonlinear elliptic partial differential equations and application to an existence result, SIAM J. Math. Anal. 23 (1992), no. 2, 326333.

[6] L. Boccardo, S. Segura de León, and C. Trombetti, Bounded and unbounded solutions for a class of quasi-linear elliptic problems with a quadratic gradient term, J. Math. Pures Appl. (9) 80 (2001), no. 9, 919-940.

[7] P. Drabek, A. Kufner, and F. Nicolosi, Nonlinear elliptic equations, singular and degenerate cases, preprint, 1996, University of West Bohemia. 
[8] J.-L. Lions, Quelques Méthodes de Résolution des Problèmes aux Limites Non Linéaires, Dunod, Paris, 1969.

[9] A. Porretta, Nonlinear equations with natural growth terms and measure data, Proceedings of the 2002 Fez Conference on Partial Differential Equations (Tex, 2002), Electron. J. Differ. Equ. Conf., vol. 9, Southwest Texas State University, 2002, pp. 183-202.

L. Aharouch: Département de Mathématiques et Informatique, Faculté des Sciences Dhar-Mahraz, BP 1796 Atlas, Fès, Morocco

E-mail address: 1_aharouch@yahoo.fr

Y. Akdim: Département de Mathématiques et Informatique, Faculté des Sciences Dhar-Mahraz, BP 1796 Atlas, Fès, Morocco

E-mail address: akdimyoussef@yahoo.fr

E. Azroul: Département de Mathématiques et Informatique, Faculté des Sciences Dhar-Mahraz, BP 1796 Atlas, Fès, Morocco

E-mail address: elzroul@caramail.com 


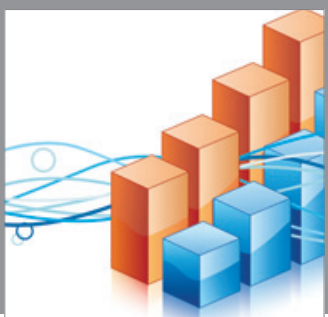

Advances in

Operations Research

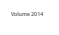

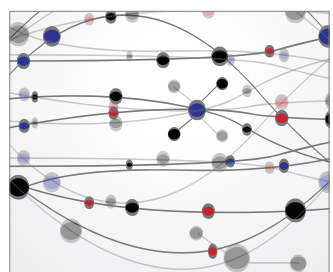

\section{The Scientific} World Journal
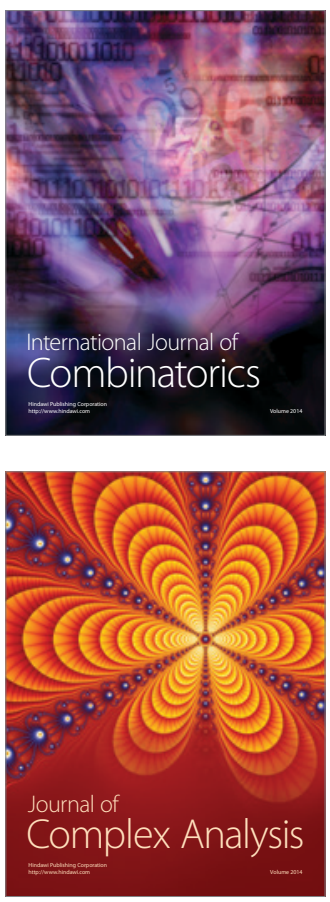

International Journal of

Mathematics and

Mathematical

Sciences
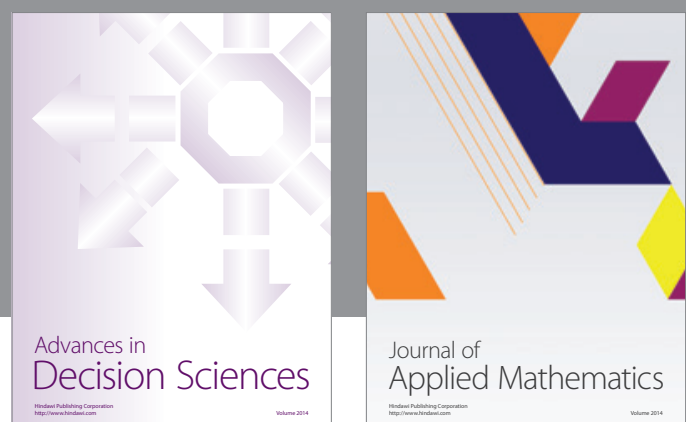

Journal of

Applied Mathematics
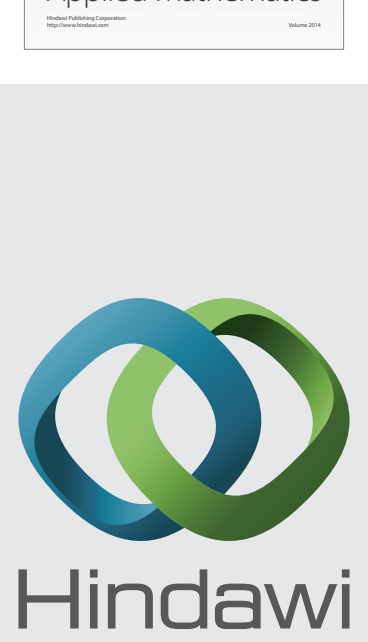

Submit your manuscripts at http://www.hindawi.com
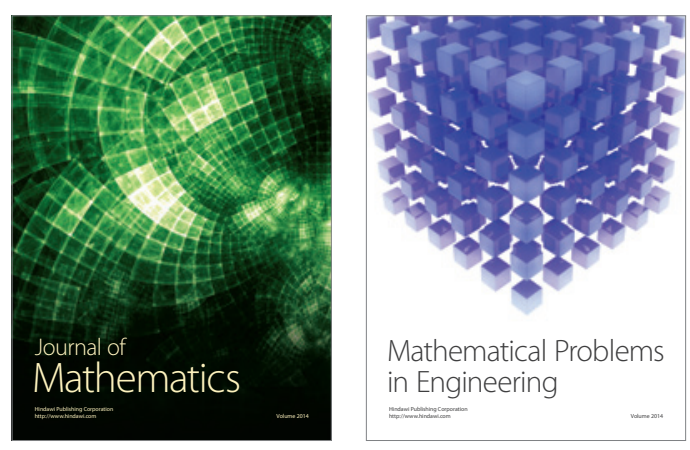

Mathematical Problems in Engineering
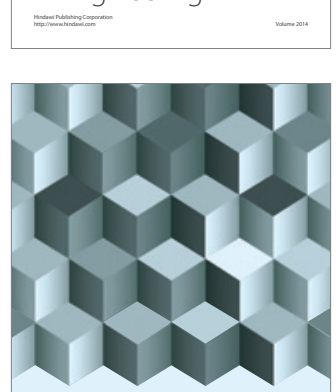

Journal of

Function Spaces
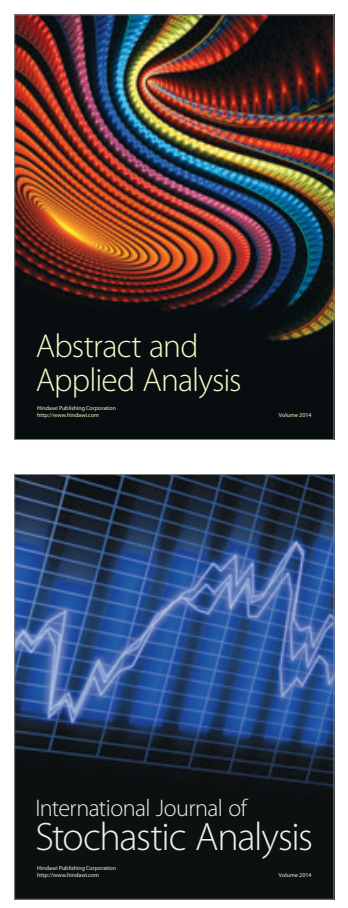

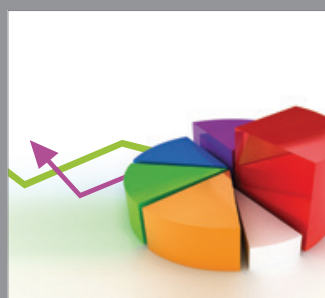

ournal of

Probability and Statistics

Promensencen
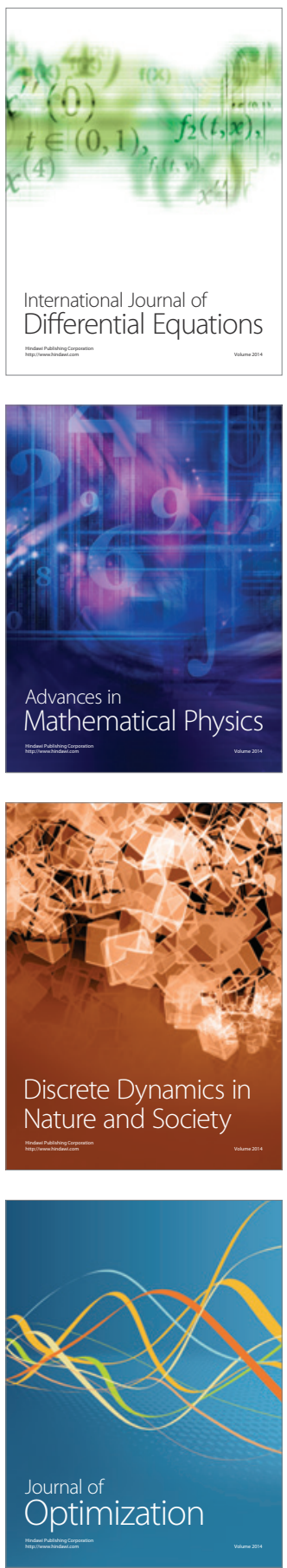